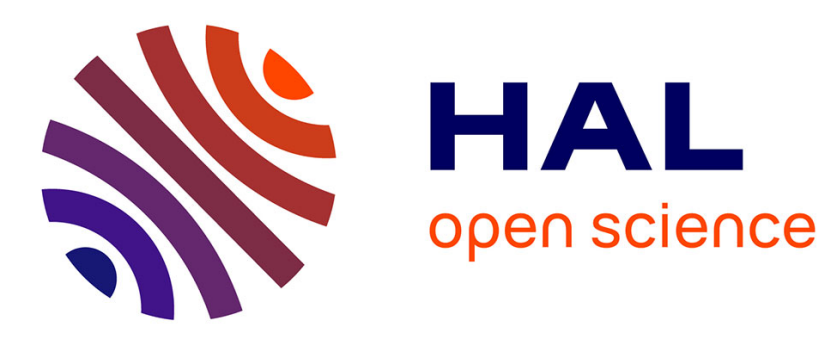

\title{
Robustness of Bioprocess Feedback Control to Biodiversity
}

\author{
Francis Mairet, Olivier A. Bernard
}

\section{To cite this version:}

Francis Mairet, Olivier A. Bernard. Robustness of Bioprocess Feedback Control to Biodiversity. AIChE Journal, 2016, pp.24. 10.1002/aic.15604 . hal-01410765

\section{HAL Id: hal-01410765 \\ https://hal.inria.fr/hal-01410765}

Submitted on 6 Dec 2016

HAL is a multi-disciplinary open access archive for the deposit and dissemination of scientific research documents, whether they are published or not. The documents may come from teaching and research institutions in France or abroad, or from public or private research centers.
L'archive ouverte pluridisciplinaire HAL, est destinée au dépôt et à la diffusion de documents scientifiques de niveau recherche, publiés ou non, émanant des établissements d'enseignement et de recherche français ou étrangers, des laboratoires publics ou privés. 


\title{
Robustness of Bioprocess Feedback Control to Biodiversity*
}

\author{
Francis Mairet and Olivier Bernard \\ Inria Biocore, BP93, 06902 Sophia-Antipolis Cedex, France
}

March 31, 2016

\begin{abstract}
The design of control laws for bioprocesses are generally based on simplified single-species models. Biodiversity (made of a mixture of different species or strains) is nonetheless inherent in any artificial ecosystem in a bioreactor. Here we propose to define and study the robustness to biodiversity of bioprocess control laws: given a control law designed for one species, what happens when additional species are present? We illustrate our approach with a well used control law which regulates substrate concentration using measurement of growth activity. Depending on the properties of the additional species, the control law can lead to the required objective, but also to an undesired monospecies equilibrium point, coexistence, or even a failure point. Finally, we show that, for this case, the robustness can be improved by a saturation of the control. Robustness to biodiversity is a difficult issue which should be better accounted for in the control design.
\end{abstract}

Topical area: Process Systems Engineering

Keyword: Biotechnology, stability analysis, nonlinear systems, bioreactor, multispecies, coexistence.

*A preliminary version of this paper was presented at the 19th IFAC World Congress (Cape Town, 2014). 


\section{Introduction}

Biodiversity is inherent in any bioprocess, even when the use of a single microorganism is initially targeted. This diversity, desired or endured, can involve a lot of different species (e.g. several hundreds of archaea and bacteria species in anaerobic digestion [1]). For monospecific cultures, different strains can be initially present or resulting from natural mutation within the process (e.g. in pharmaceutical biotechnology). Nevertheless, theoretical developments in automatic control for bioprocesses are generally based on the single-species dogma, i.e. one macroscopic reaction involves one species (see e.g. $[2,3,4]$ ). A few works have shed light on the importance of biodiversity for the modelling and control of bioprocesses. For example, the effect of multispecies has been shown in simulation for anaerobic digestion [5]. It has also been tackled in the development of optimal strategies (via simulation for the start-up of an anaerobic digester [6] and analytically for fed-batch operation [7]).

Classically, robustness of control laws are considered with respect to parameter uncertainties or noise measurements (see e.g. $[8,9,10,4]$ ), but to our knowledge, never to the presence of other species having their own dynamics. Indeed, given a control law designed for one species, what happens when two or more species are actually present? To tackle this problem, we introduce the concept of robustness to biodiversity. A control law is said to be robust to bio-

diversity for a given set of species if the performance of the control law (in a sense that will be defined later) is not reduced by the presence of any species from this set.

To define the robustness to biodiversity of a control law, one should study the asymptotic behavior of a system including not only a single species, but an assemblage of species competing for a substrate. This has been widely done in mathematical ecology in order to study the competition of species $[11,12]$. When a constant dilution rate is applied, the principle of competitive exclusion for Monod-type models, states that at most one species will survive. On the other 
hand, $[13,14,15]$ have proposed control laws in order to obtain coexistence. Finally, recent developments have been proposed for the selection of species $[16,17,18]$, with the objective to design a control strategy in order to select a species of interest. These papers propose some useful tools which will be used to study robustness to biodiversity.

After defining the concept of robustness to biodiversity, we illustrate our approach with a control law proposed in [9] in order to regulate substrate concentration. Finally, we propose a modification of the control law in order to increase its robustness.

\section{Framework and definition}

We consider a generic model of micro-organism growth limited by one substrate in a chemostat [3]. Denote $x_{1}$ and $s$ the concentrations of biomass and substrate respectively. The chemostat model is given by:

$$
\left\{\begin{array}{l}
\dot{s}=u(t)\left(s_{i n}-s\right)-k_{1} \mu_{1}(s) x_{1} \\
\dot{x_{1}}=\left(\mu_{1}(s)-u(t)\right) x_{1}
\end{array}\right.
$$

where $u(t)$ is the dilution rate (which will be our control signal), $\mu_{1}(s)$ the specific growth rate, $s_{i n}$ the input substrate concentration, and $k_{1}$ the pseudo yield coefficient.

A species is defined by a parameter vector $\theta \in S \subset \mathbb{R}_{+}^{n_{\theta}}$, gathering stoechiometric and kinetic parameters (i.e. parameters of the growth rate $\mu$ ). We will refer to $S$ as a set of species.

Denoting $\xi$ the state vector, we consider a control law $u(\xi)$ which globally stabilizes System (1) towards a set-point $\xi^{*}=\left(s^{*},\left(s_{i n}-s^{*}\right) / k_{1}\right)$ associated to a scalar productivity criterion $\mathcal{P}(\xi)$ (often maximising this criterion). For example, $\mathcal{P}(\xi)$ can be related to the biomass productivity at the reactor outlet $\left(\mathcal{P}(\xi)=u x_{1}\right)$ or to the substrate removal rate $\left(\mathcal{P}(\xi)=u\left(s_{\text {in }}-s\right)\right)$. Note that the productivity is often zero for $u=0$ or for the species washout $\left(x_{1}=0\right.$ and 
$\left.s=s_{i n}\right)$

By definition, the robustness to biodiversity of this control law guarantees that, in presence of multispecies, the system will be stabilized at a point $\xi$ with the same or better productivity $\mathcal{P}(\xi)$ than for the set-point in monoculture $\xi^{*}$.

Definition 1. The control law $u(\xi)$ is said to be $(S, n, \mathcal{P})$ robust iff, for any $n$ species $x_{i}, i=2, \ldots, n+1$ such that $\theta_{i} \in S$, the control law globally stabilizes the system:

$$
\left\{\begin{array}{l}
\dot{s}=u(\xi)\left(s_{i n}-s\right)-\sum_{i=1}^{n+1} k_{i} \mu_{i}(s) x_{i} \\
\dot{x_{i}}=\left(\mu_{i}(s)-u(\xi)\right) x_{i}, \quad i=1,2, \ldots, n+1,
\end{array}\right.
$$

towards a point $\hat{\xi}$ such that $\mathcal{P}(\hat{\xi}) \geq \mathcal{P}\left(\xi^{*}\right)$, where $\xi^{*}=\left(s^{*},\left(s_{\text {in }}-s^{*}\right) / k_{1}, 0, \ldots, 0\right)$.

\section{A didactic example}

To highlight the concept of robustness to biodiversity, we consider the control law proposed in [9]. This must be seen as a simple didactic example to introduce the ideas and show their relevance for bioprocess management.

\subsection{Control law design}

In the following, we assume the following hypotheses on the growth rate and the measurement:

Hypothesis 1. (i) The specific growth rates $\mu_{i}$ are nonnegative functions $\mathcal{C}^{1}$ of $s$ with $\mu_{i}(0)=0$.

(i) The measurement of total growth is available:

$$
y(\xi)=\sum_{i=1}^{n+1} l_{i} \mu_{i}(s) x_{i}
$$

where $l_{i}$ is the associated yield coefficient for species $i$.

In anaerobic digestion, the methane flow rate measurement is directly related to the growth rate, given the low solubility of methane (see e.g. $[9,19]$ ). 
For other bioprocesses, the total growth can often be estimated using observerbased estimator $[20,21]$ using for example the measurement of gaseous $\mathrm{O}_{2}$ or $\mathrm{CO}_{2}$.

Given a set-point $s^{*} \in\left(0, s_{i n}\right)$, we consider the feedback law proposed by [9]:

Theorem 1. Under Hypotheses 1, the feedback control law

$$
u(\xi)=\gamma y(\xi)
$$

with $\gamma=\frac{k_{1}}{l_{1}\left(s_{i n}-s^{*}\right)}$ globally stabilizes System (1) towards the positive set point $\left(s^{*}, x_{1}^{*}\right)$, where $x_{1}^{*}=\left(s_{\text {in }}-s^{*}\right) / k_{1}$.

Proof. See [9].

To illustrate our presentation, we will consider the productivity criterion defined as the treated organic load (for waste-water treatment):

$$
\mathcal{P}(\xi)=u(\xi)\left(s_{\text {in }}-s\right)
$$

\subsection{Adding another species}

Robustness of Control law (3) with respect to fluctuations in $s_{i n}$ has been considered in [19]. Here, we will study its robustness to biodiversity. For sake of simplicity, we restrict our analyses to the presence of only one additional species. Recalling that the dynamics of $M:=s+\sum k_{i} x_{i}$ is given by

$$
\dot{M}=u(\xi)\left(s_{i n}-M\right)
$$

we additionally consider initial conditions $\left(s, x_{1}, x_{2}\right)$ in the attractive invariant manifold $\left\{\left(s, x_{1}, x_{2}\right) \mid s+k_{1} x_{1}+k_{2} x_{2}=s_{i n}\right\}$. Under this hypothesis, System 
(2) with Control law (3) becomes:

$$
\dot{x_{i}}=\left[\mu_{i}(s)-\gamma \sum_{j=1}^{2} l_{j} \mu_{j}(s) x_{j}\right] x_{i}, \quad i=1,2 .
$$

with $s=s_{i n}-\sum_{i=1}^{2} k_{i} x_{i}$.

System (5) admits the following equilibria:

- Substrate depletion $(s=0): E_{0}=\left\{\left(x_{1}^{0}, x_{2}^{0}\right) \in \mathbb{R}_{+}^{2} \mid k_{1} x_{1}^{0}+k_{2} x_{2}^{0}=s_{\text {in }}\right\}$.

This corresponds to an equilibrium line.

- Washout $\left(s=s_{i n}\right): E_{w}=(0,0)$.

- Monospecies $1(s=s *): E_{1}=\left(x_{1}^{*}, 0\right)$, where $x_{1}^{*}=\frac{1}{\gamma l_{1}}$

- Monospecies $2: E_{2}=\left(0, x_{2}^{*}\right)$, where $x_{2}^{*}=\frac{1}{\gamma l_{2}}$ iff

$$
\frac{k_{2}}{l_{2}}<\gamma s_{i n}
$$

The substrate concentration at equilibrium is $s=s_{2}^{*}:=s_{i n}-\frac{k_{2}}{l_{2} \gamma}$. Note that $(\mathrm{C} 1)$ guarantees that $s_{2}^{*}>0$.

- Coexistence : this equilibrium point is possible if the two growth functions have an intersection point:

$$
\exists s_{c} \in\left(0, s_{i n}\right) \mid \mu_{1}\left(s_{c}\right)=\mu_{2}\left(s_{c}\right) .
$$

$E_{c}=\left(\tilde{x}_{1}, \tilde{x}_{2}\right)$ exists iff $\tilde{x}_{1}$ and $\tilde{x}_{2}$ are positive, i.e. iff:

$$
\tilde{x}_{1}=\frac{l_{2}\left(s_{c}-s_{2}^{*}\right)}{k_{2} l_{1}-k_{1} l_{2}}>0 \quad \text { and } \quad \tilde{x}_{2}=\frac{l_{1}\left(s_{c}-s^{*}\right)}{k_{1} l_{2}-k_{2} l_{1}}>0 .
$$

These conditions impose that the substrate concentrations of monospecies equilibria are located on either side of the intersection point of the growth functions:

$$
\text { - if } \frac{l_{2}}{k_{2}}>\frac{l_{1}}{k_{1}} \text {, then } s^{*}<s_{c}<s_{2}^{*} \text {. }
$$


- if $\frac{l_{1}}{k_{1}}>\frac{l_{2}}{k_{2}}$, then $s_{2}^{*}<s_{c}<s^{*}$ (recalling that $s_{2}^{*} \leq 0$ if $E_{2}$ does not exist).

For each equilibrium $E_{\bullet}$, we denote $\mathcal{P}_{\bullet}$ the corresponding productivity.

\subsection{Local stability analysis}

The Jacobian matrix $J$ of System (5) in the invariant manifold is:

$$
J=\left[\begin{array}{cc}
B_{1}-\gamma\left(y(\xi)+x_{1} A_{1}\right) & -k_{2} \mu_{1}^{\prime}(s) x_{1}-\gamma x_{1} A_{2} \\
-k_{1} \mu_{2}^{\prime}(s) x_{2}-\gamma x_{2} A_{1} & B_{2}-\gamma\left(y(\xi)+x_{2} A_{2}\right)
\end{array}\right]
$$

where $A_{i}=l_{i} \mu_{i}(s)-k_{i}\left(l_{1} \mu_{1}^{\prime}(s) x_{1}+l_{2} \mu_{2}^{\prime}(s) x_{2}\right)$ and $B_{i}=\mu_{i}(s)-k_{i} \mu_{i}^{\prime}(s) x_{i}$.

\subsubsection{Local stability of $E_{0}$.}

The eigenvalues of the Jacobian matrix $J$ evaluated at $E_{0}$ are $\lambda_{1}=0$ and

$$
\lambda_{2}=\mu_{1}^{\prime}(0) x_{1}^{0}\left(\gamma s_{i n} l_{1}-k_{1}\right)+\mu_{2}^{\prime}(0) x_{2}^{0}\left(\gamma s_{i n} l_{2}-k_{2}\right) .
$$

Given that $\gamma s_{i n} l_{1}>k_{1}, E_{0}$ is unstable if $\gamma s_{i n} l_{2}>k_{2}$, i.e. if $E_{2}$ exists (C1). If the second term is negative, recalling that $E_{0}=\left\{\left(x_{1}^{0}, x_{2}^{0}\right) \in \mathbb{R}_{+}^{2} \mid k_{1} x_{1}^{0}+k_{2} x_{2}^{0}=s_{\text {in }}\right\}$, there will be a subset of $E_{0}$ where $\lambda_{2}<0$, which corresponds to locally stable equilibria.

\subsubsection{Stability of $E_{w}$.}

At $E_{w}$, the Jacobian matrix $J$ becomes:

$$
J=\left[\begin{array}{cc}
\mu_{1}\left(s_{i n}\right) & 0 \\
0 & \mu_{2}\left(s_{i n}\right)
\end{array}\right]
$$

so $E_{w}$ is unstable. 


\subsubsection{Local stability of $E_{1}$ and $E_{2}$.}

The eigenvalues of the Jacobian matrix $J$ evaluated at $E_{1}$ are $\lambda_{1}=-\mu_{1}\left(s^{*}\right)$ and $\lambda_{2}=\mu_{2}\left(s^{*}\right)-\mu_{1}\left(s^{*}\right)$. Thus, $E_{1}$ is locally stable iff

$$
\mu_{1}\left(s^{*}\right)>\mu_{2}\left(s^{*}\right)
$$

Similarly, $E_{2}$ is locally stable iff

$$
\mu_{2}\left(s_{2}^{*}\right)>\mu_{1}\left(s_{2}^{*}\right)
$$

\subsubsection{Local stability of $E_{c}$.}

At the equilibrium point $E_{c}$, the trace and determinant of $J$ writes:

$$
\begin{aligned}
\operatorname{tr}(J) & =-\mu_{1}\left(s_{c}\right)-\gamma \tilde{x}_{1} \tilde{x}_{2}\left[\mu_{2}^{\prime}\left(s_{c}\right)-\mu_{1}^{\prime}\left(s_{c}\right)\right]\left(k_{2} l_{1}-k_{1} l_{2}\right) \\
\operatorname{det}(J) & =\mu_{1}\left(s_{c}\right) \gamma \tilde{x}_{1} \tilde{x}_{2}\left[\mu_{2}^{\prime}\left(s_{c}\right)-\mu_{1}^{\prime}\left(s_{c}\right)\right]\left(k_{2} l_{1}-k_{1} l_{2}\right)
\end{aligned}
$$

Thus, $E_{c}$ is locally stable iff

$$
\left[\mu_{2}^{\prime}\left(s_{c}\right)-\mu_{1}^{\prime}\left(s_{c}\right)\right]\left(k_{2} l_{1}-k_{1} l_{2}\right)>0 .
$$

\subsection{Robustness to biodiversity of Control law (3)}

We now consider the following assumption on the growth functions:

Hypothesis 2. The specific growth rates $\mu_{i}(s)$ are assumed to be of Monod type $\mu_{i}(s)=\bar{\mu}_{i} \frac{s}{s+K_{i}}$, where $\bar{\mu}_{i}$ and $K_{i}$ are respectively the maximum growth rate and the half-saturation constant.

Remark that only the ratio of the yield coefficients $\alpha_{i}:=l_{i} / k_{i}$ matters to compute local stability and productivity at steady state. As a consequence, a species can be defined by the set of three parameters $\theta_{i}=\left(\bar{\mu}_{i}, K_{i}, \alpha_{i}\right)$, and we 

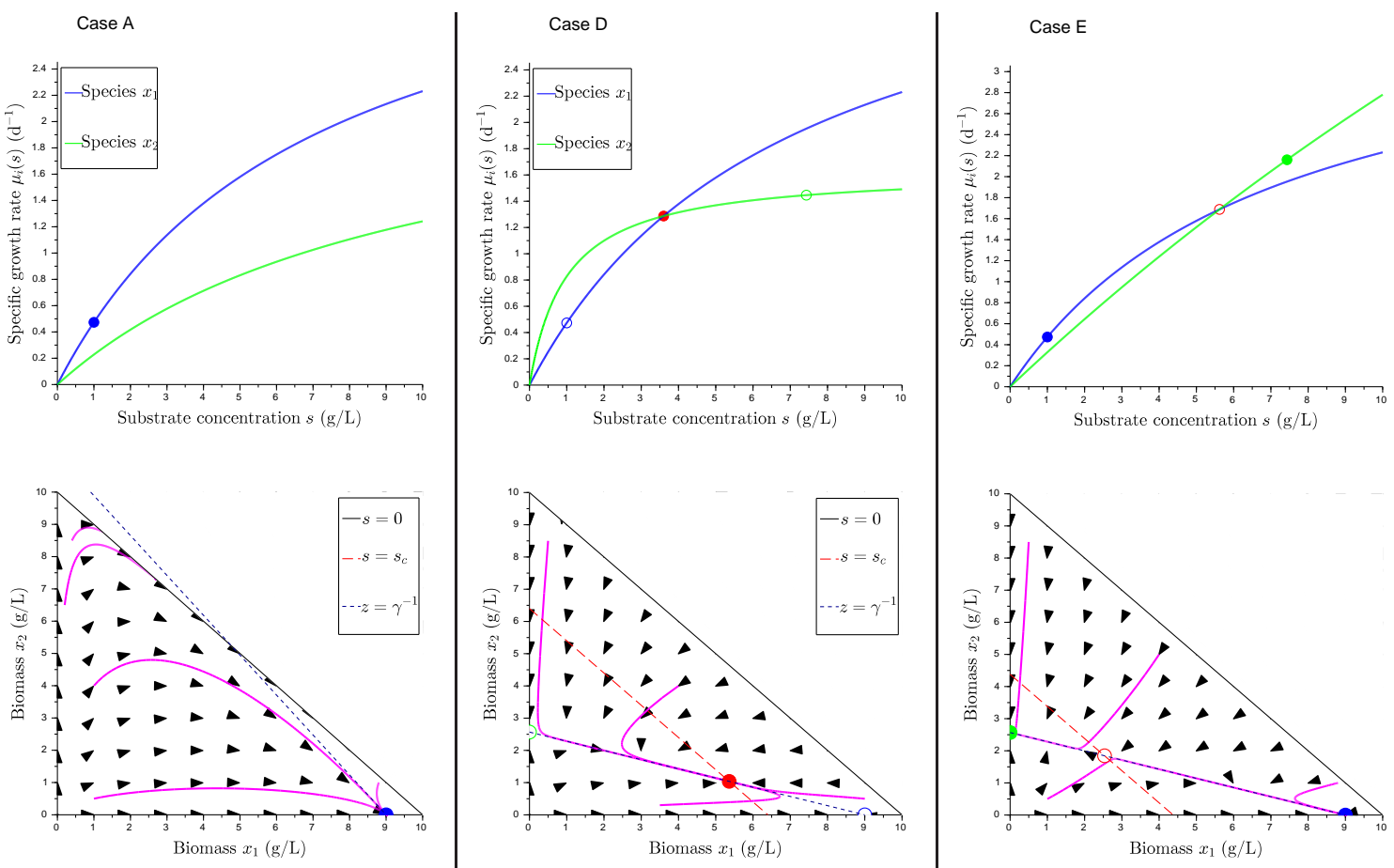

Substrate concentration $s(\mathrm{~g} / \mathrm{L})$

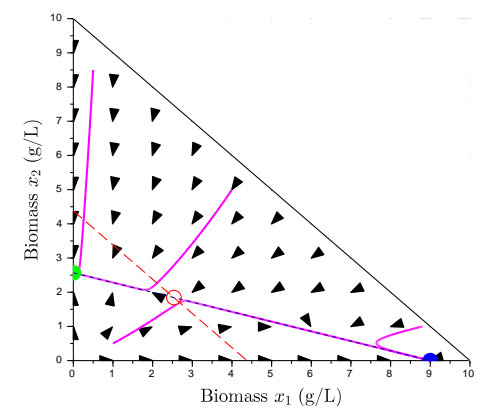

Figure 1: Asymptotic behavior of System (5), Case A, D, and E (see Proposition 1 and Table 1). Top: Specific growth rates as a function of substrate concentration. Bottom: Phase portraits with some trajectories (in purple). Filled circle: stable equilibria, open circles: unstable equilibria. Blue: species $x_{1}$, green: species $x_{2}$, red: coexistence. 
consider

$$
S:=\left[\bar{\mu}^{-}, \bar{\mu}^{+}\right] \times\left[K^{-}, K^{+}\right] \times\left[\alpha^{-}, \alpha^{+}\right] .
$$

Now, we can define the largest $\operatorname{set}^{1} S_{R} \subset S$ such that Control law (3) is $\left(S_{R}, 1, \mathcal{P}\right)$ robust.

Proposition 1. Given initial conditions $\left(x_{1}^{0}, x_{2}^{0}\right) \in \mathbb{R}_{+}^{* 2}$ such that $k_{1} x_{1}^{0}+k_{2} x_{2}^{0}<$ $s_{\text {in }}$ (i.e. $s^{0}>0$ ), the asymptotic behavior of System (5) is given in Table 1.

Proof. First, note that System (5) is dissipative: all positive trajectories lie in the bounded set $\left\{\left(x_{1}, x_{2}\right) \in \mathbb{R}_{+}^{2} \mid k_{1} x_{1}+k_{2} x_{2} \leq s_{i n}\right\}$.

If (C1) does not hold, a subspace of $E_{0}$ is locally stable (so the control law is not robust to any $\mathcal{P}$ which cancels when $u$ cancels, and in particular for (4)).

Now, we assume that $(\mathrm{C} 1)$ holds, and we consider the subset $\{\theta \in S$ | $\left.\gamma s_{i n} \alpha_{2}>1\right\}$. Let $z=l_{1} x_{1}+l_{2} x_{2}$. In the coordinates $\left(z, x_{1}\right)$, System (5) becomes:

$$
\left\{\begin{array}{l}
\dot{z}=y(\xi)(1-\gamma z) \\
\dot{x_{1}}=\left(\mu_{1}(s)-\gamma y(\xi)\right) x_{1}
\end{array}\right.
$$

Given that $E_{0}$ and $E_{w}$ are repulsive, $y(t)$ cannot tend to zero, and thus, $z$ tends to $\gamma^{-1}$, which shows the absence of periodic solutions or cycles. Therefore, any trajectory converges to an equilibrium point. The previous studies of equilibria and local stability allow us to conclude for the different cases.

The asymptotic behavior of System (5) is illustrated on Fig. 1 for different cases. The control law is robust whenever all the stable equilibria lead to a productivity greater or equal to $\mathcal{P}_{1}$ (see Table 1 and Appendix).

\subsection{Results}

In order to restrict our analysis to a more realistic case, we consider a subset of $S$ more relevant from an ecological point of view. We consider that each parameter represents a trait that is involved in the fitness of the species. A

\footnotetext{
${ }^{1}$ note that we ignore subsets of null measure corresponding to non-generic cases, since they will never appear in practice (for example the cases $s^{*}=s_{c}$, or $s_{2}^{*}=s_{c}$ ).
} 
Table 1: Equilibria and robustness for Control law (3) - System (5) (see Proposition 1)

\begin{tabular}{cccccccc}
\hline Case & Conditions & $E_{0}$ & $E_{w}$ & $E_{1}$ & $E_{2}$ & $E_{c}$ & Robustness $^{c}$ \\
\hline A & $\neg$ C1 & LS & uns & - & no & - & $\mathrm{F}$ \\
B & C1.C4. $(\neg$ C5) & uns & uns & GAS & uns & no & T \\
C & C1. $(\neg C 4) . C 5$ & uns & uns & uns & GAS & no & T iff $\mathcal{P}_{2} \geq \mathcal{P}_{1}$ \\
D & C1. $(\neg C 4) .(\neg \text { C5 })^{a}$ & uns & uns & uns & uns & GAS & T iff $\mathcal{P}_{c} \geq \mathcal{P}_{1}$ \\
E & C1.C4.C5 $5^{b}$ & uns & uns & LS & LS & uns & T iff $\mathcal{P}_{2} \geq \mathcal{P}_{1}$
\end{tabular}

LS: locally stable, GAS:globally stable, uns: unstable, -:whatever, T/F: True/False.

a: or equivalently C1.C2.C3.C6, b: or equivalently C1.C2.C3.( $\neg$ C6), c: see Appendix

species with the best values for each trait will outcompete all the other species in any environmental conditions, and such super mutant should not exist. Actually, one may assume trade-offs between the different traits. For $m$ traits, we consider that the $m$ archetypes (or specialist, i.e. a species with the best value for one trait and the worst values for the others) define a Pareto front where all the species lie [22]. In our example, we consider three archetypes $x_{\bar{\mu}}, x_{K}, x_{\alpha}$, with $\theta_{\bar{\mu}}=\left(\bar{\mu}^{+}, K^{+}, \alpha^{-}\right), \theta_{K}=\left(\bar{\mu}^{-}, K^{-}, \alpha^{-}\right)$, and $\theta_{\alpha}=\left(\bar{\mu}^{-}, K^{+}, \alpha^{+}\right)$. Note that the best value for $K$ is $K^{-}$. These archetypes define the subset $\bar{S}$ :

$$
\bar{S}:=\left\{\theta=a \theta_{\bar{\mu}}+b \theta_{K}+c \theta_{\alpha}, \forall(a, b, c) \in[0,1]^{3} \mid a+b+c=1\right\}
$$

Thus, for any triplet $\left(a_{i}, b_{i}, c_{i}\right) \in[0,1]^{3} \mid a_{i}+b_{i}+c_{i}=1$, the parameter vector of the species $x_{i}\left(a_{i}, b_{i}, c_{i}\right)$ is given by:

$$
\theta_{i}=\left(\begin{array}{c}
a_{i} \bar{\mu}^{+}+\left(1-a_{i}\right) \bar{\mu}^{-} \\
b_{i} K^{-}+\left(1-b_{i}\right) K^{+} \\
c_{i} \alpha^{+}+\left(1-c_{i}\right) \alpha^{-}
\end{array}\right)
$$

The morphospace, i.e. the space of trait values, is represented on a ternary plot in Figure 2. Each vertex of the triangle represents an archetype. We consider Control law (3) designed for a species $x_{1}(0.3,0.4,0.3)$ located near the center of the morphospace. After discretizing the morphospace, we test for each additional species which conditions $(\mathrm{C} 1, \mathrm{C} 4, \mathrm{C} 5)$ hold in order to determine 
the asymptotic behavior of the system (see Proposition 1 and Table 1). This allows us to define $S_{R}$ (represented in color on Figure 2), a subset of $\bar{S}$, such that Control law $(3)$ is $\left(S_{R}, 1, \mathcal{P}\right)$ robust. First, one can see that the presence of an additional species can increase the productivity. On the other hand, the control law is not robust for a large subsets of $\bar{S}$, corresponding mainly to an additional species with a smaller yield coefficient than species $x_{1}\left(\alpha_{2}<\alpha_{1}\right)$. Two situations may occur: the productivity at a stable equilibrium point $\left(E_{2}\right.$ or $E_{c}$ ) is smaller than the productivity $\mathcal{P}_{1}$ (the white area), or there is a reactor shutdown ( $s$ and $y$ converge towards zero, the black area). A small decrease in productivity can actually be tolerated (although it is not considered in the present definition of robustness to biodiversity). On the other hand, the reactor shutdown is much more problematic and represents a real drawback of Control law (3). This motivates new control law design in order to avoid as far as possible such situation, i.e. to increase the robustness to biodiversity of the control law.

\section{Robustification of the control law}

\subsection{Saturated control law}

In order to avoid reactor shutdown, we propose to saturate the dilution rate when growth activity is low. The feedback law proposed by [9] becomes:

$$
u(\xi)=\left\{\begin{array}{cc}
\gamma y(\xi) & \text { if } y(\xi)>\underline{y} \\
\gamma \underline{y} & \text { if } y(\xi) \leq \underline{y}
\end{array}\right.
$$

where the design parameter $\underline{y}$ must verify:

$$
\underline{y}<\frac{\min \left\{\mu_{1}\left(s_{i n}\right), \mu_{1}\left(s^{*}\right)\right\}}{\gamma} .
$$

This condition guaranties that the washout is repulsive, and that there is no non-trivial equilibrium with $y \leq \underline{y}$. 

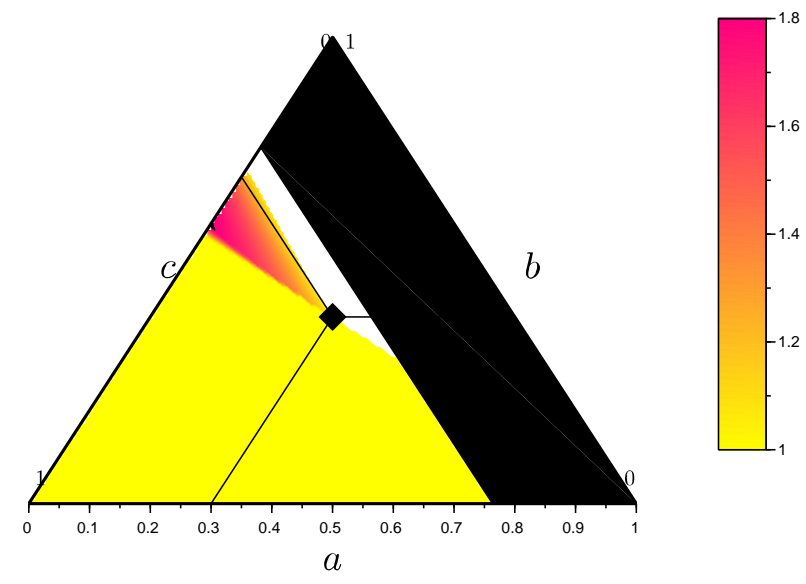

Figure 2: Robustness of Control law (3) on a ternary plot of the morphospace $\bar{S}$. Each vertex represents an archetype: $x_{\bar{\mu}}(1,0,0), x_{K}(0,1,0), x_{\alpha}(0,0,1)$. The diamond represents species $x_{1}(0.3,0.4,0.3)$. The set of species $S_{R}$, such that Control law (3) is $\left(S_{R}, 1, \mathcal{P}\right)$ robust, is represented in color. The color map represents the relative increase of productivity (with respect to $\mathcal{P}_{1}$ ). The black area corresponds to reactor shutdown (Case A). 
Theorem 2. Under Hypotheses 1, the feedback control law (8) globally stabilizes System (1) towards the positive set point $\left(s^{*}, x_{1}^{*}\right)$.

Proof. Consider $M=s+k_{1} x_{1}$ whose dynamics is:

$$
\dot{M}=u(\xi)\left(s_{i n}-M\right)
$$

It follows that the set $\Omega=\left\{(s, x) \in \mathbb{R}_{+}^{2} \mid M \leq \max \left\{M(0), s_{i n}\right\}\right\}$ is positively invariant. In open loop with a constant dilution rate $D<\mu_{1}\left(s_{i n}\right)$, it is well known that System (1) has a non-trivial equilibrium point which is globally asymptotically stable (GAS)[11]. Given Condition (9), for a constant dilution rate $\gamma \underline{y}$, the system has a GAS equilibrium $\left(\underline{s},\left(s_{i n}-\underline{s}\right) / k_{1}\right)$, where $\underline{s}$ is the smallest root of $\gamma \underline{y}=\mu_{1}(s)$. At this point, we have:

$$
y\left(\underline{s},\left(s_{i n}-\underline{s}\right) / k_{1}\right)=\alpha_{1} \gamma \underline{y}\left(s_{i n}-\underline{s}\right)=\underline{y} \frac{s_{i n}-\underline{s}}{s_{i n}-s^{*}} .
$$

Given that $\underline{s}<s^{*}$, we get $y\left(\underline{s},\left(s_{i n}-\underline{s}\right) / k_{1}\right)>\underline{y}$, so this point is located outside the set $\underline{\Omega}=\{(s, x) \in \Omega \mid y(\xi) \leq \underline{y}\}$. Thus, under Control law (8), all trajectories with initial conditions in $\underline{\Omega}$ will leave this set. Now, in $\bar{\Omega}:=\Omega \backslash \underline{\Omega}$, System (1) rewrites:

$$
\left\{\begin{array}{l}
\dot{s}=\gamma y(\xi)\left(s^{*}-s\right) \\
\dot{x_{1}}=\gamma y(\xi)\left(x_{1}^{*}-x\right) .
\end{array}\right.
$$

Given that $y(\xi)$ is lower bounded and that $y\left(s^{*}, x_{1}^{*}\right)>\underline{y},\left(s^{*}, x_{1}^{*}\right)$ belongs to $\bar{\Omega}$ and it is the unique stable equilibrium point in this set. Thus, System (1) under Control law (8) has a unique stable equilibrium point $\left(s^{*}, x_{1}^{*}\right)$ in the positively invariant set $\Omega$. Equation (10) eliminates the possibility of periodic orbit, so Poincaré-Bendixson theorem allows to conclude that $\left(s^{*}, x_{1}^{*}\right)$ is GAS.

The global stability of the control law relies on the transition between regions: every trajectory starting in $\underline{\Omega}$ will enter in $\bar{\Omega}$. Such transitions between regions where a constant dilution rate is applied has supported the design of an hybrid control law in [23] where quantized measurements $y$ are considered. 


\subsection{Stability analysis with multispecies}

We will now characterize how the saturation of the control law increases its robustness to biodiversity. In line with Section 3, we consider the presence of one additional species with initial conditions $\left(s, x_{1}, x_{2}\right)$ in the attractive invariant manifold $\left\{\left(s, x_{1}, x_{2}\right) \mid s+k_{1} x_{1}+k_{2} x_{2}=s_{i n}\right\}$. Given that the saturation level $\underline{y}$ is typically small, we assume for sake of simplicity that $\gamma \underline{y}<\mu_{2}\left(s_{i n}\right)$. We divide the positively invariant set $\Lambda=\left\{\xi \in \mathbb{R}_{+}^{2} \mid k_{1} x_{1}+k_{2} x_{2} \leq s_{\text {in }}\right\}$ into two regions: $\underline{\Lambda}:=\{\xi \in \Lambda \mid y(\xi) \leq \underline{y}\}$ and $\bar{\Lambda}:=\Lambda \backslash \underline{\Lambda}$. We get the following system:

$$
\dot{x_{i}}=\left(\mu_{i}(s)-u(\xi)\right) x_{i}, \quad i=1,2 .
$$

with $s=s_{i n}-\sum_{i=1}^{2} k_{i} x_{i}$ and $u(\xi)=\left\{\begin{array}{cc}\gamma y(\xi) & \text { if } \xi \in \bar{\Lambda} \\ \gamma \underline{y} & \text { if } \xi \in \underline{\Lambda}\end{array}\right.$

For $\bar{\Lambda}$, the existence and stability of the equilibria defined in Section 3 remain unchanged whenever they belong to this set, i.e. this concerns $E_{1}$, and respectively $E_{2}$ and $E_{c}$ iff :

$$
y\left(0, x_{2}^{*}\right)>\underline{y},
$$

and

$$
y\left(\tilde{x}_{1}, \tilde{x}_{2}\right)>\underline{y} .
$$

Note that $E_{0}$ does not exist anymore. Now in $\underline{\Lambda}$, recalling the classical results of microbial competition in the chemostat [11], we can state that the washout $E_{w}$ is unstable given that $u=\gamma \underline{y}<\mu_{1}\left(s_{i n}\right)$. Additionally, one equilibrium with only species $x_{2}$ can exist. Let $\underline{s_{2}}$ the root of $\gamma \underline{y}=\mu_{2}(s)$, with $0<\underline{s_{2}}<s_{i n}$. The equilibrium $\underline{E_{2}}=\left(0, \underline{x_{2}}\right)$, where $\underline{x_{2}}=\left(s_{i n}-\underline{s_{2}}\right) / k_{2}$, exists if $y\left(0, \underline{x_{2}}\right) \leq \underline{y}$, or equivalently, if $(\mathrm{C} 7)$ does not hold. It is locally stable iff:

$$
\mu_{2}\left(\underline{s_{2}}\right)>\mu_{1}\left(\underline{s_{2}}\right) .
$$

We now want to characterize the global behavior of the system. 
Lemma 1. For $\gamma \underline{y}<\mu_{2}\left(s_{2}^{*}\right)$, all trajectories of System (12) converge towards an equilibrium point.

Proof. Givent that $\gamma \underline{y}<\mu_{2}\left(s_{2}^{*}\right)$, we get:

$$
y\left(0, x_{2}^{*}\right)=l_{2} \mu_{2}\left(s_{2}^{*}\right) x_{2}^{*}=\frac{\mu_{2}\left(s_{2}^{*}\right)}{\gamma}>\underline{y},
$$

and, whenever $E_{c}$ exists:

$$
y\left(\tilde{x}_{1}, \tilde{x}_{2}\right)=\frac{\mu_{1}\left(s_{c}\right)}{\gamma}>\frac{\min \left\{\mu_{1}\left(s_{1}^{*}\right), \mu_{2}\left(s_{2}^{*}\right)\right\}}{\gamma}>\underline{y},
$$

so $E_{2}$ and $E_{c}$ (if it exists) belong to $\bar{\Lambda}$.

Recalling $z(\xi)=l_{1} x_{1}+l_{2} x_{2}$, consider the function $V(\xi)=\frac{1}{2}\left(z(\xi)-\gamma^{-1}\right)^{2}$. In $\bar{\Lambda}$, we have:

$$
\dot{V}(\xi)=-\gamma y(\xi)\left(z(\xi)-\gamma^{-1}\right)^{2} \leq 0 .
$$

In $\underline{\Lambda}$, if $z(\xi)>\gamma^{-1}$, we obtain:

$$
\begin{aligned}
\dot{V}(\xi) & =(y(\xi)-\gamma \underline{y} z(\xi))\left(z(\xi)-\gamma^{-1}\right) \\
& \leq \underline{y}(1-\gamma z(\xi))\left(z(\xi)-\gamma^{-1}\right) \\
& \leq-\gamma \underline{y}\left(z(\xi)-\gamma^{-1}\right)^{2} \leq 0 .
\end{aligned}
$$

Let $z_{m}=\max _{\xi \in \underline{\Lambda} \mid z(\xi)<\gamma^{-1}} z(\xi)$. For any $z_{c} \in\left(z_{m} ; \gamma^{-1}\right)$, we can deduce that the set $\Lambda_{c}=\left\{\xi \in \Lambda \mid z(\xi) \geq z_{c}\right\}$ is positively invariant. Let

$$
S=\left\{\xi \in \Lambda_{c} \mid \dot{V}(\xi)=0\right\}=\left\{\xi \in \Lambda_{c} \mid z(\xi)=\gamma^{-1}\right\}
$$

No solution can stay identically in $S$, other than the equilibria $E_{1}, E_{2}$, or $E_{c}$ (if it exists). Applying the LaSalle theorem, every solution starting in $\Lambda_{c}$ approaches one of these equilibrium points as $t \rightarrow+\infty$. Finally, in $\Lambda \backslash \Lambda_{c}$, there is only one equilibrium $E_{w}$, which is a repeller located on the boundary. This eliminates the possibility of periodic or homoclinic orbit, so any solution starting in $\Lambda$ will 
Table 2: Equilibria and robustness for Control law (8) - System (12)

\begin{tabular}{|c|c|c|c|c|c|c|c|}
\hline Case & Conditions & $E_{w}$ & $E_{1}$ & $E_{2}$ & $\underline{E_{2}}$ & $E_{c}$ & Robustness $^{a}$ \\
\hline$\overline{B^{\prime}}$ & C4.(ᄀC5).C7 & $\overline{\text { uns }}$ & GAS & no or uns & no or uns & no & $\mathrm{T}$ \\
\hline $\mathrm{C}^{\prime}$ & $(\neg \mathrm{C} 4) . \mathrm{C} 5 . \mathrm{C} 7$ & uns & uns & GAS & no & no & $\mathrm{T}$ iff $\mathcal{P}_{2} \geq \mathcal{P}_{1}$ \\
\hline C" & $(\neg \mathrm{C} 4) . \mathrm{C} 5 .(\neg \mathrm{C} 7)$ & uns & uns & no & GAS & no & $\mathrm{T}$ iff $\underline{\mathcal{P}_{2}} \geq \mathcal{P}_{1}$ \\
\hline$D^{\prime}$ & $(\neg \mathrm{C} 4) \cdot(\neg \mathrm{C} 5) . \mathrm{C} 8$ & uns & uns & no or uns & no or uns & GAS & $\mathrm{T}$ iff $\overline{\mathcal{P}_{c}} \geq \mathcal{P}_{1}$ \\
\hline D" & $(\neg \mathrm{C} 4) .(\neg \mathrm{C} 5) .(\neg \mathrm{C} 8)$ & uns & uns & no & GAS & no & $\mathrm{T}$ iff $\underline{\mathcal{P}_{2}} \geq \mathcal{P}_{1}$ \\
\hline E' & C4.C5. $(\neg \mathrm{C} 8)$ & uns & GAS & no & uns & no & $\overline{\mathrm{T}}$ \\
\hline E" & C4.C5.C7 & uns & LS & LS & no & uns & $T$ iff $\mathcal{P}_{2} \geq \mathcal{P}_{1}$ \\
\hline E"' & C4.C5. $(\neg \mathrm{C} 7) \mathrm{C} 8$ & uns & LS & no & LS & uns & $\mathrm{T}$ iff $\mathcal{P}_{2} \geq \mathcal{P}_{1}$ \\
\hline
\end{tabular}

converge towards an equilibrium points, either $E_{1}, E_{2}$, or $E_{c}$.

From extensive numerical simulations, the lemma seems to hold also for $\gamma \underline{y}>\mu_{2}\left(s_{2}^{*}\right)$. In the following, we will assume that it is true, so all trajectories of System (12) converge towards an equilibrium point. For Monod growth rates (Hypothesis 2), we can deduce the global behavior of System (12), given in Table 2. The saturation of the control has increased the robustness of the control law, see Fig. 3 in comparison with Fig. 2. In particular, reactor shutdown - the most problematic case - does not occur anymore. The analytic study of robustness for $n$ species is obviously more delicate and is currently under investigation.

\section{Simulation study}

We illustrate our approach with numerical simulations. System (2) was simulated under Control laws (3) and (8). The number of species $n$, the species characteristics $\theta_{i} \in \bar{S}$ and the initial conditions $\xi(0)$ were drawn randomly. In Figure 4, we show an example where Control law (3) leads to a reactor shutdown, while the trajectory converges towards the working equilibrium point with Control law (8). We have shown previously that the saturation of the control law allows to increase the robustness for one additional species with initial conditions in the invariant set. Our simulations tends to show that the new control law is actually robust for $n>2$ additional species with any initial 


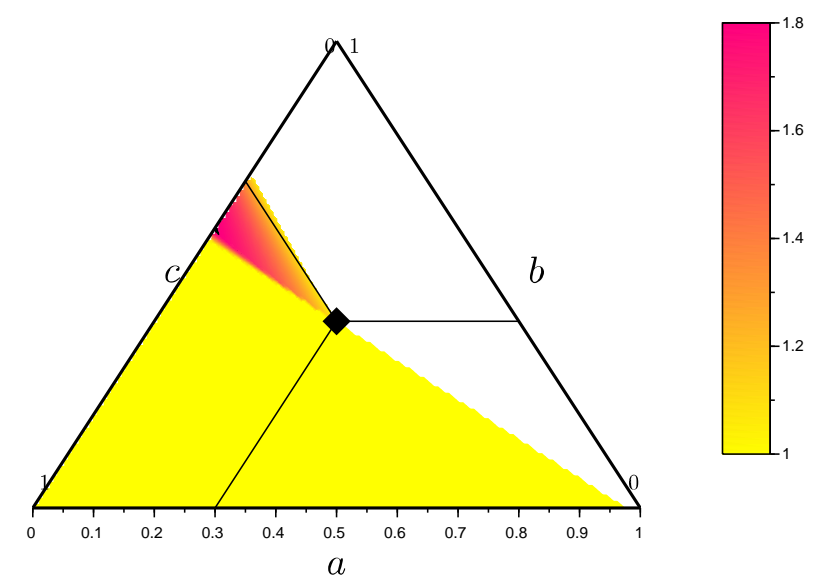

Figure 3: Robustness of Control law (8) on a ternary plot of the morphospace $\bar{S}$. The saturation of the control allows to increase the robustness and to avoid reactor shutdown. Same legend as Fig.2.

conditions. Finally, Figure 5 illustrates the case where the additional species allows to increase the productivity of the system.

\section{Discussion}

The robustness of control strategies with respect to parametric uncertainty has been classically studied for bioprocesses. Here, we have defined the robustness to biodiversity. These two approaches are clearly not equivalent. Actually, the $n$ species can be seen as one species with time-varying kinetic and stoechiometric parameters. Such time-varying aspect is generally not considered in parametric uncertainty. Moreover, the robustness to biodiversity also involves species competition/selection/coexistence. These phenomena, which affect bioprocess productivity, are not considered in the robustness with respect to parametric uncertainty. 

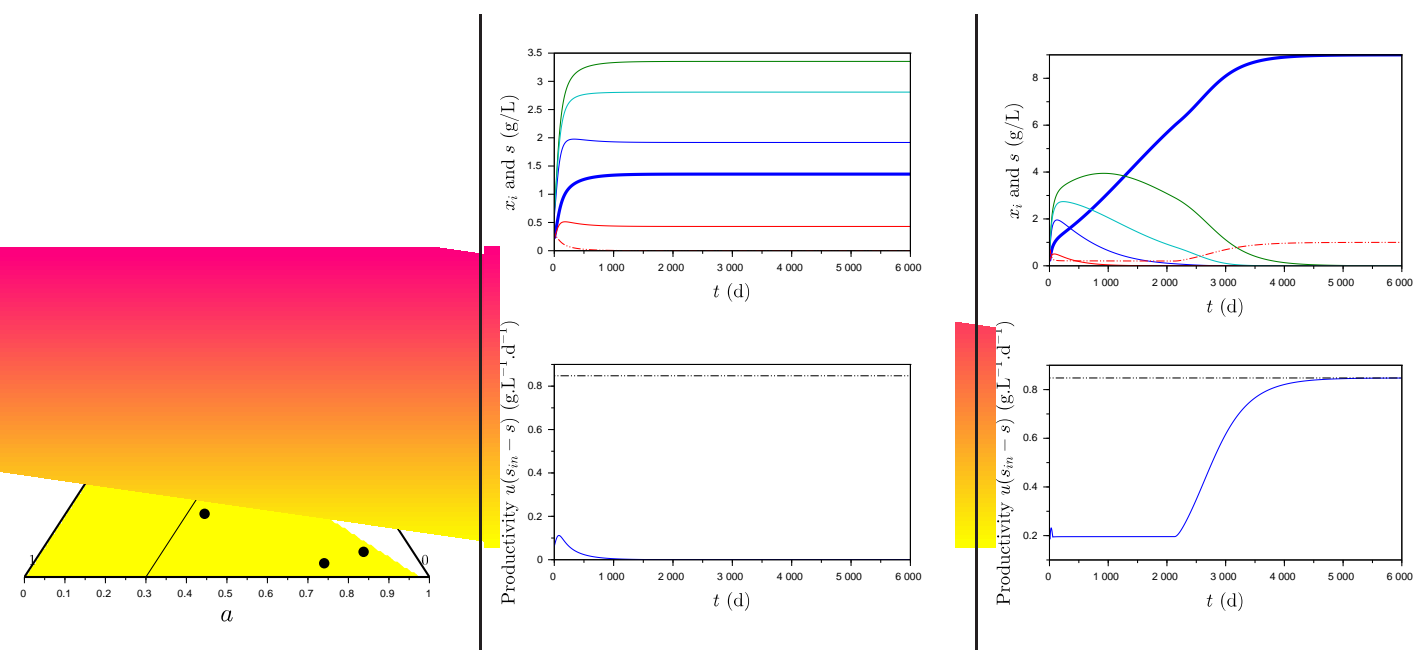

Figure 4: Simulation of System (2) without (middle) and with (right) saturation of the control law (Eq. (3) and (8) respectively). Top: Blue thick line: species $x_{1}$, thin lines : species $x_{i}, i>1$, red dashed line: substrate $s$. Bottom: productivity (blue line) and set-point (black dashed line). Left: ternary plot of the morphospace $\bar{S}$ (see legend of Fig. 2). The black dots represent the additional species drawn randomly in the robust set of Control law (8).
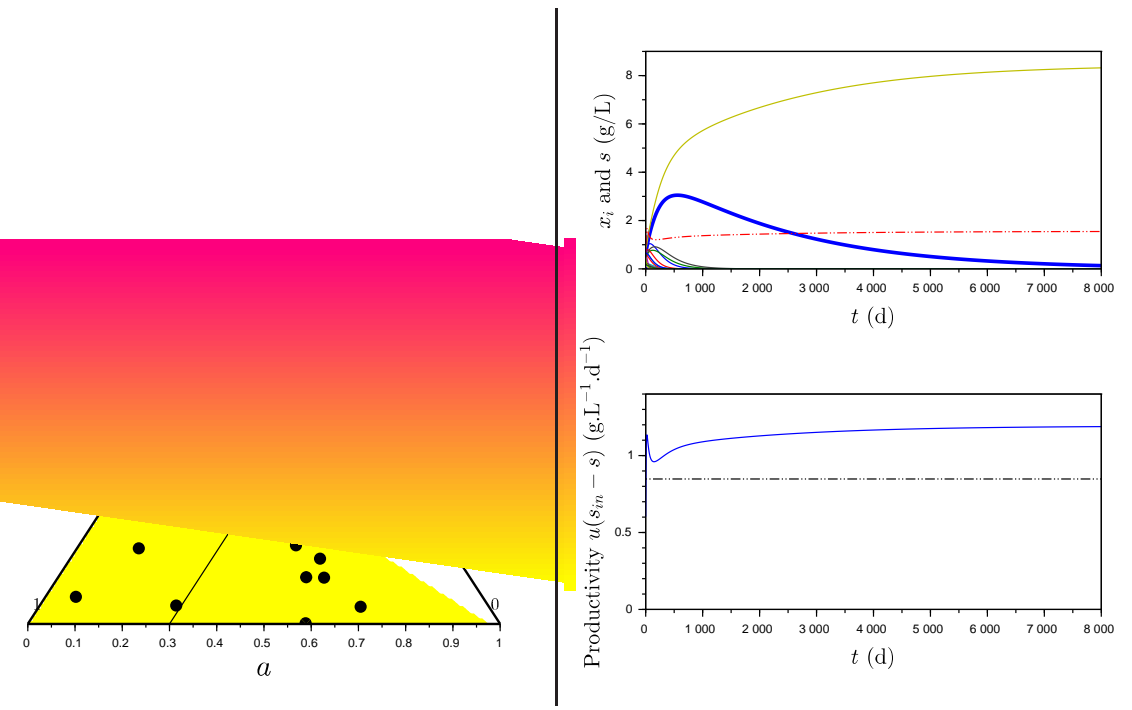

Figure 5: Simulation of System (2) with Control law (8) for another set of species and initial conditions. Same legend as Fig. 4. One additional species outcompetes species $x_{1}$ and allows to improve the productivity. 


\subsection{Link with species competition}

The classical theory of species competition [11] does not apply when the chemostat is operated with a closed loop control, and some surprises can arise. For example, if we consider a slow growing species $x_{2}$ (with $\mu_{2}(s)<\mu_{1}(s), \forall s \in$ $\left(0, s_{i n}\right)$ ), we expect that this species will rapidly be outcompeted (as it happens in open loop), so it should not affect the asymptotic behavior of the system. Actually, with Control law (3), the substrate depletion equilibrium $E_{0}$ can become locally stable in presence of $x_{2}$ (if $\alpha_{2} \gamma s_{i n}<1$ ), so this species can cause reactor shutdown (see Figure 1, Case A).

\subsection{Coexistence of two species}

The stable coexistence of two species limited by one substrate is possible whenever their specific growth rates intersect. In open loop, this is not feasible in practice since the dilution should be chosen exactly equal to the rate at which growth curves intersect. On the other hand, [13] and [14] have proposed feedback controls for the stable coexistence of two species, based on measurement of biomass concentrations. Here, we have shown that the feedback law (3) can also lead to stable coexistence, whenever the set-point $s^{*}$ is chosen accordingly. Assuming without loss of generality $\mu_{1}^{\prime}\left(s_{c}\right)>\mu_{2}^{\prime}\left(s_{c}\right)$, the coexistence point $E_{c}$ is globally asymptotically stable iff $\alpha_{1}<\alpha_{2}$ and $0<s^{*}<s_{c}<s_{2}^{*}<s_{\text {in }}$ (see Fig. 1 , case D). If $\alpha_{1}>\alpha_{2}$, Control law (3) does not generate stable coexistence.

\subsection{Challenges}

Maintaining a high bioreactor productivity despite invasive species (or new individuals resulting from natural mutations) turns out to be a challenging problem to ensure process robustness. New questions arise now, such as detecting and preventing the apparition of "bad species". Observers could be set-up to early identify new competing species with reduced productivity capability. Control strategies, such as extremum-seeking [8], could be adapted in order to consider 
multispecies and maintain conditions favorable for the settlement of enhanced species with productivity increase. As demonstrated in this paper, such control strategies could use this opportunity to continuously improve the reactor performance.

\section{Conclusion}

We have introduced the concept of robustness to biodiversity for bioprocess control laws. We have illustrated our approach with a control law proposed in [9]. Depending on the characteristics of the additional species, some counterintuitive results may appear such as coexistence, or even reactor shutdown when a slow-growing species is introduced. The saturation of the control law allows to avoid this phenomena, and so it increases the robustness. As demonstrated in our example, this framework can be used to design robust control laws and therefore better tame biodiversity within a biotechnological process.

\section{Appendix}

We give the conditions on parameters $\theta_{2}$ that should be fulfilled to avoid any productivity loss at alternative steady states (see Tables 1 and 2). For $E_{2}$, this yields:

$$
\mathcal{P}_{2} \geq \mathcal{P}_{1} \Leftrightarrow \bar{\mu}_{2} \frac{\alpha_{2} \gamma s_{i n}-1}{\alpha_{2}\left[\alpha_{2} \gamma\left(K_{2}+s_{i n}\right)-1\right]} \geq \bar{\mu}_{1} \frac{s^{*}}{\alpha_{1}\left(K_{1}+s^{*}\right)}
$$

For $E_{c}$, given that $s_{c}=\frac{\bar{\mu}_{1} K_{2}-\bar{\mu}_{2} K_{1}}{\bar{\mu}_{2}-\bar{\mu}_{1}}$, we have:

$$
\mathcal{P}_{c} \geq \mathcal{P}_{1} \Leftrightarrow \frac{\left(\bar{\mu}_{1} K_{2}-\bar{\mu}_{2} K_{1}\right)\left[s_{i n}\left(\bar{\mu}_{2}-\bar{\mu}_{1}\right)-\bar{\mu}_{1} K_{2}+\bar{\mu}_{2} K_{1}\right]}{\left(K_{2}-K_{1}\right)\left(\bar{\mu}_{2}-\bar{\mu}_{1}\right)} \geq \bar{\mu}_{1} \frac{s^{*}\left(s_{i n}-s^{*}\right)}{K_{1}+s^{*}}
$$

For $\underline{\mathcal{P}_{2}}$, using $\underline{s_{2}}=\frac{K_{2} \gamma \underline{y}}{\bar{\mu}_{2}-\gamma \underline{y}}$, we finally get:

$$
\underline{\mathcal{P}_{2}} \geq \mathcal{P}_{1} \Leftrightarrow \underline{y} \frac{s_{i n} \bar{\mu}_{2}-\gamma \underline{y}\left(K_{2}+s_{i n}\right)}{\bar{\mu}_{2}-\gamma \underline{y}} \geq \alpha_{1} \bar{\mu}_{1} \frac{s^{*}\left(s_{i n}-s^{*}\right)^{2}}{K_{1}+s^{*}}
$$




\section{References}

[1] Rivière D., Desvignes V., Pelletier E., et al. Towards the definition of a core of microorganisms involved in anaerobic digestion of sludge The ISME journal. 2009;3:700-714.

[2] Vera Julio, Torres Néstor V, Moles Carmen G, Banga Julio. Integrated nonlinear optimization of bioprocesses via linear programming AIChE journal. 2003;49:3173-3187.

[3] Dochain D.. Automatic control of bioprocesses. John Wiley \& Sons, Inc. 2008.

[4] Dimitrova Neli, Krastanov Mikhail. Nonlinear adaptive control of a model of an uncertain fermentation process International Journal of Robust and Nonlinear Control. 2010;20:1001-1009.

[5] Ramirez I., Volcke E., Rajinikanth R., Steyer J.-P.. Modeling microbial diversity in anaerobic digestion through an extended ADM1 model Water research. 2009;43:2787-2800.

[6] Sbarciog M., Vande Wouwer A.. Some Considerations About Control of Multi-species Anaerobic Digestion Systems in Proceedings of the 7th International Conference on Mathematical Modelling (MATHMOD)(Vienna, Austria) 2012.

[7] Gajardo P., Ramirez C. H., Rapaport A.. Minimal time sequential batch reactors with bounded and impulse controls for one or more species SIAM Journal on Control and Optimization. 2008;47:2827-2856.

[8] Zhang Tao, Guay Martin, Dochain Denis. Adaptive extremum seeking control of continuous stirred-tank bioreactors AIChE journal. 2003;49:113123.

[9] Mailleret L., Bernard O., Steyer J.-P.. Robust Nonlinear Adaptive Control for Bioreactors with Unknown Kinetics Automatica. 2004;40:8:365-383. 
[10] Gouze Jean-Luc, Robledo Gonzalo. Robust control for an uncertain chemostat model International Journal of Robust and Nonlinear Control. 2006;16:133-155.

[11] Smith H. L., Waltman P.. The theory of the chemostat: dynamics of microbial competition. Cambridge University Press 1995.

[12] Grognard Frédéric, Masci Pierre, Benoît Eric, Bernard Olivier. Competition between phytoplankton and bacteria: exclusion and coexistence Journal of mathematical biology. 2015;70:959-1006.

[13] De Leenheer P., Smith H.. Feedback control for chemostat models Journal of Mathematical Biology. 2003;46:48-70.

[14] Gouzé J.-L., Robledo G.. Feedback control for nonmonotone competition models in the chemostat Nonlinear Analysis: Real World Applications. 2005;6:671-690.

[15] Mazenc F., Malisoff M., Harmand J.. Further results on stabilization of periodic trajectories for a chemostat with two species IEEE TAC. 2008;53:6674.

[16] Mairet F., Muñoz-Tamayo R., Bernard O.. Driving Species Competition in a Light-limited Chemostat in 9th IFAC Symposium on Nonlinear Control Systems (NOLCOS) 2013.

[17] Bayen T., Mairet F.. Optimization of the separation of two species in a chemostat Automatica. 2014;50:1243-1248.

[18] Mairet Francis, Bernard Olivier. The photoinhibistat: operating microalgae culture under photoinhibition for strain selection in Proceedings of the 11th International Symposium on Dynamics and Control of Process Systems (DYCOPS) 2016.

[19] Karafyllis I., Kravaris C., Syrou L., Lyberatos G.. A vector Lyapunov function characterization of input-to-state stability with application to ro- 
bust global stabilization of the chemostat European Journal of Control. 2008;14:47-61.

[20] Bastin G., Dochain D.. On-line estimation and adaptive control of bioreactors. New York: Elsevier 1990.

[21] Perrier M., Azevedo S. Feyo, Ferreira E.C., Dochain D.. Tuning of observerbased estimators: theory and application to the on-line estimation of kinetic parameters Control Engineering Practice. 2000;8:377-388.

[22] Shoval O, Sheftel H, Shinar G, et al. Evolutionary trade-offs, Pareto optimality, and the geometry of phenotype space Science. 2012;336:1157-1160.

[23] Mairet F., Gouzé J.-L.. Hybrid Control of a Bioreactor with Quantized Measurements IEEE Transactions on Automatic Control. 2015. To appear. 